\title{
Can Traditional Breathing Methods Reduce Stress?
}

\section{Tateyuki Morisawa1, Mayumi Watanabe ${ }^{1 *}$, Hidetoshi Mori1,2, Kazushi Nishijo², Kazuhide Tomita ${ }^{3}$, Kenta Kawamura ${ }^{3}$, Jun Sato', Nozomu Mandai1}

\author{
${ }^{1}$ Information Science Center for Humanities and Sciences School of Health Sciences, Ibaraki Prefectural University of Health \\ Sciences, Ibaraki, Japan \\ ${ }^{2}$ Faculty of Health Sciences, National University Corporation Tsukuba University of Technology, Ibaraki, Japan \\ ${ }^{3}$ Department of Physical Therapy, Ibaraki Prefectural University of Health Sciences, Ibaraki, Japan \\ Email: *watanabem62@gmail.com
}

How to cite this paper: Morisawa, T., Watanabe, M., Mori, H., Nishijo, K., Tomita, K., Kawamura, K., Sato, J. and Mandai, N. (2020) Can Traditional Breathing Methods Reduce Stress? Health, 12, 923-931. https://doi.org/10.4236/health.2020.128069

Received: July 15, 2020

Accepted: August 9, 2020

Published: August 12, 2020

Copyright (c) 2020 by author(s) and Scientific Research Publishing Inc. This work is licensed under the Creative Commons Attribution International License (CC BY 4.0).

http://creativecommons.org/licenses/by/4.0/

\begin{abstract}
Purpose: Traditional medicines have unique breathing methods and they are widely believed that they are good for health promotion and good for relax. However, there is little evidence. The purpose of this study is to discuss if such specific breathings could effectively reduce stress and be able to help to reach a relaxed state in the body as well as in the mind. To objectively understand the state of stress/relax, we assessed the condition of the autonomic nervous system (ANS) as well as heart rate (HR) and body temperature (BT), which instantly reflect ANS. Methods: Fourteen healthy male volunteers participated in this study and we investigated four kinds of breathing with them: Natural breathing (Control), Abdominal Pressure breathing (Pressure), Abdominal breathing (Abdominal) and Reverse Abdominal breathing (Reverse). Results: Only Control group showed increase of HF/decrease of HR, showing increase parasympathetic nervous system, which indicates the condition of relax. On the hand, all experimental groups of unique breathings showed increase of sympathetic nervous system, meaning stress. Discussion: Our hypothesis was that traditional breathing methods would decrease stress, however, our results were the total opposite to it. This reason might be found: 1) our subjects might be too young and naive, 2) they did not have enough training or experience, and 3) enough and appropriate duration of breathings was needed. Therefore, we consider that appropriate program and enough training time to use to specific breathing are needed to obtain the effects of traditional breathing.
\end{abstract}

\section{Keywords}

Breathing, Autonomic Nervous System (ANS), Body Temperature (BT), Stress, 
Heart Rate (HR)

\section{Introduction}

The world health organization released ICD-11 in June 2018, and classified traditional medicines including yoga or traditional Chinese medicine [1]. Such traditional medicines put place a high priority on breathing, yoga or Taiqi, for example. They believed specific that breathing methods are good for health promotion; however, there are little evidence.

A specific method of breathing is also emphasized among zen monks or budo of samurai warriors. For instance, they considered that Tanden breathing method, a kind of abdominal breathing, can reduce stress, support concentration and lead to relax. Few physicians studied this method and they found it a breathing method with abdominal pressure including abdominal breathing (abdominal pressure rises at inhalation) and reverse abdominal breathing (abdominal pressure rises at exhalation) [2] [3] [4]. However, such traditional methods are often handed down from mouth to ear, from a teacher to his/her student. Its esoteric knowledge is still unknown.

Physical therapists focused on changes in the transverses abdominis muscle ( $\operatorname{Tr} A)$ thickness and showed that training of the $\operatorname{TrA}$, which is rarely activated on a daily base, utilizing breathing with abdominal pressure can achieve strengthening of TrA contraction [5]. Researchers of sports and urology had tried to study in various ways [6] [7] [8] [9] [10]. However, their physical approach did not refer to the mental effects of breathing. As a result, the secret to relax is not revealed.

The purpose of this study is to discuss if such specific breathings could effectively reduce stress and be able to help to reach a relaxed state in the body as well as in the mind. To objectively understand the state of stress / relax, we assessed the condition of the autonomic nervous system (ANS) and body temperature (BT).

ANS has two divisions: the sympathetic nervous system (SNS) and parasympathetic nervous system (PNS). Under stress, alpha adrenergic stimulus induces SNS dominance [11] [12] [13] [14]. At the same time, such stress induces fight-or-flight response as W B Cannon confirmed [15] [16]. To fight/flight whole body muscles and brain needs more oxygen; therefore, ANS increases heart rate (HR). As stress instantaneously contracts vessels, BT simultaneously decreases [13] [14] [17]. On the other hand, HR reflects the status of ANS via facilitation of cardiac vagal activity and cardiac sympathetic nerve [18] [19]. Thus, we may consider HR and BT are new indicators of ANS status.

Therefore, we assessed those indicators to evaluate stress/relax status in this study. And then, we discussed the change induced by such specific breathings. In short, the hypothesis of this study is if traditional breathing methods increase 
PNS (decrease SNS), can reduce stress and help to reach relax.

\section{Methods}

\subsection{Participants}

Fourteen healthy male volunteers (average $21.0 \pm 0.5$ years old) participated in this study. We prepared a recruitment poster to explain the purpose and procedures of the study. To meet the inclusion criteria of the study, subjects needed to be healthy males with no known history of any major physical and psychological illnesses. Students with impaired vision or those who were taking any autonomic drugs were excluded.

Written informed consent was obtained from all patients and this study was approved by the Medical Ethics Committee of Ibaraki Prefectural University of Health Sciences (Ibaraki, Japan, 887-R010704).

The experiments were conducted in a room with the temperature set at $25.6^{\circ} \mathrm{C}$ $\pm 0.5^{\circ} \mathrm{C}, 42.3 \% \pm 6.7 \%$ relative humidity throughout the experimental sessions.

\subsection{Breathing Methods}

In this study we investigated four kinds of breathing; natural breathing (Control), Abdominal Pressure breathing (Pressure), Abdominal breathing (Abdominal) and Reverse Abdominal breathing (Reverse). Each distinction of breathing is illustrated referring to the previous study [2] (Figure 1).

Subjects took more than 10 minutes rest sitting in armchairs before the experiment. And then, they inhale 5 seconds and then exhale 10 seconds in each breathing.

\subsection{Measurements}

\subsubsection{HR (Heart Rate), ANS (Autonomic Nervous System) (LF, HF and LF/HF)}

We measured and recorded heart rate (HR) using electrocardiograph (LRR-03, GMS Co., Ltd., Tokyo, Japan) with disposal electrodes (Nihon Kohden Corp., Tokyo, Japan) and the measuring equipment (MP36, Zero C Seven, Inc., Tokyo, Japan).

And then, we calculated to analyze those results with analysis program (MemCalc/Tarawa, GMS Co., Ltd., Tokyo, Japan) following maker's operating instructions. Thus we obtained Low-frequency (LF, $0.04-0.15 \mathrm{~Hz}$ ), High-frequency (HF, $0.15 \sim 0.45 \mathrm{~Hz}$ ) and LF/HF ratio.

LF is comprised of rhythms with periods between 7 and $25 \mathrm{~s}$ and is affected by breathing from $\sim 3$ to $9 \mathrm{bpm}$. Within a 5 min sample, there are 12 - 45 complete periods of oscillation [20]. On the other hand, HF is influenced by breathing from 9 to $24 \mathrm{bpm}$ [21]. The ratio of LF to HF power (LF/HF) may estimate the ratio between sympathetic nervous system (SNS) and parasympathetic nervous system (PNS) activity of autonomic nervous system (ANS) [22].

However, actually many researchers consider that LF is still very controversial 


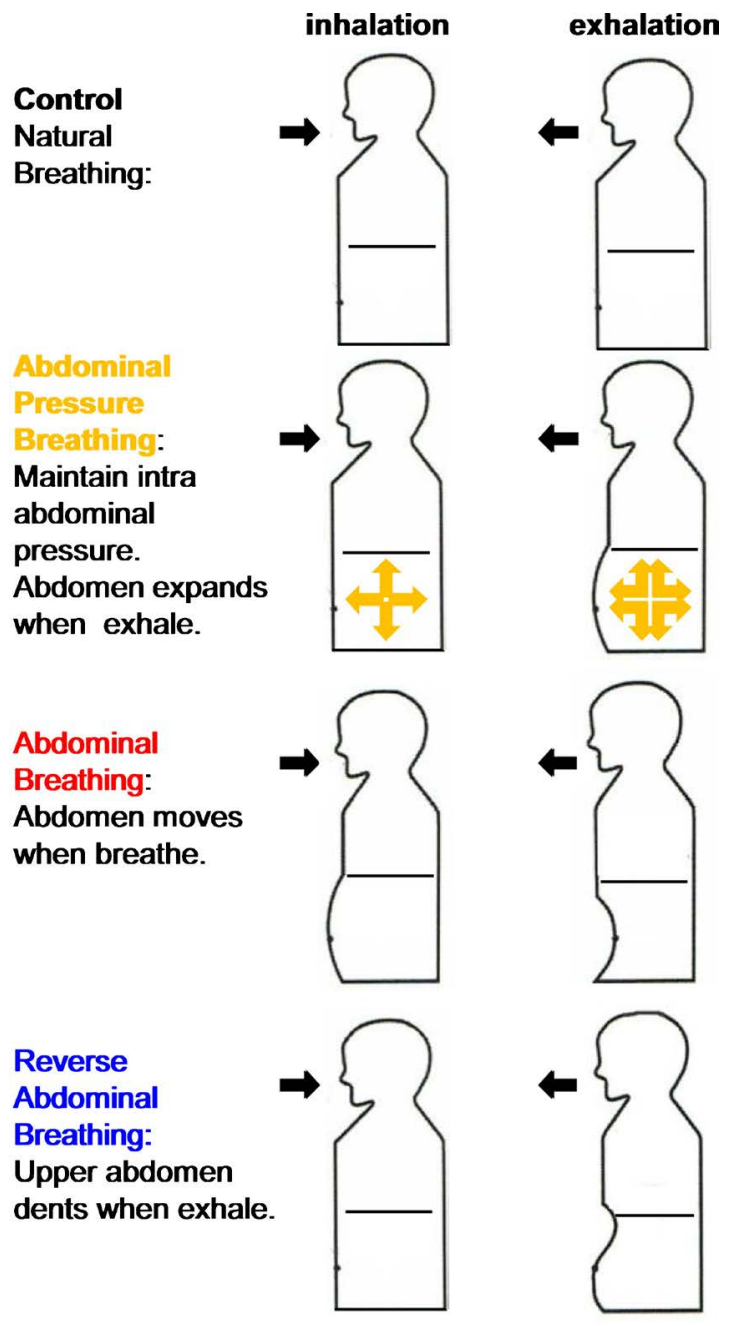

Figure 1. Breathing techniques of this study: Natural breathing (Control), Abdominal breathing (Abdominal) and Reverse Abdominal breathing (Reverse) as illustrate referring to Dr. Kubota T's study.

as an indicator of ANS status. Therefore, in this study we measure LF value, however, we do not discuss it in this study. We make use of LF only to obtain LF/HF to understand SNS status as previous studies [20] [22] [23].

\subsubsection{Body Temperature (BT)}

To measure body temperature (dorsal surface of the right/left hand) we used an infrared thermographic instrument (H2600, Nippon Avionics Co., ltd., Tokyo, Japan) and its specific program (InfReC Analyzer NS9500 Professional, Nippon Avionics Co., ltd., Tokyo, Japan) according to the vendor's instructions.

\subsection{Statistical Analysis}

Statistical analysis. Two-way ANOVA was performed for the analysis followed by a Scheffe post hoc analysis as required $(\mathrm{p}<0.05)$. SPSS Advanced Models, version 15 , software was used for the statistical analysis. The level of significance was set at $\mathrm{p}<0.05$. The values generated were mean and standard deviation (SD). 


\section{Results}

\subsection{LF (Low-Frequency)}

LF significantly increased in Control group at Post $10(\mathrm{p}=0.026)$ compared to Pre. At the same time, in Abdominal group and Reverse group showed significant increase at Post $10(\mathrm{p}=0.001)$ and $(\mathrm{p}=0.016)$ respectively. However, only Pressure group did not indicate such change (Figure 2(a)).

\subsection{HF (High-Frequency)}

Only Control group showed a significant increase of HF increase at Post 10 ( $\mathrm{p}=$ 0.047) compared to Pre. Other groups did not show such significant difference (Figure 2(b)).

\subsection{LF/HF Ratio}

Only in Reverse group, LF/HF ratio significantly increased at post 10 ( $\mathrm{p}=0.028$ ). All other groups did not show any significant difference. (Figure 2(c)).
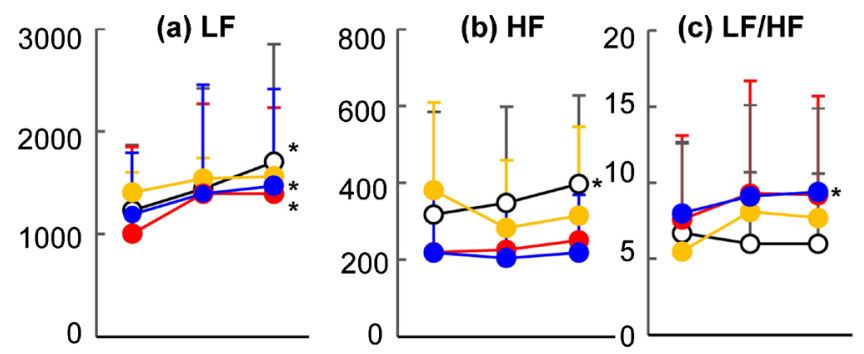

(d) HR (/min)

(e)
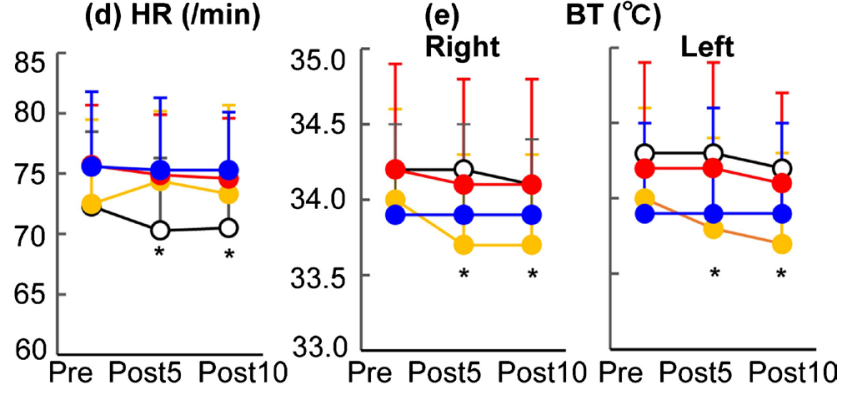

\section{(f) Visualized image of BT}

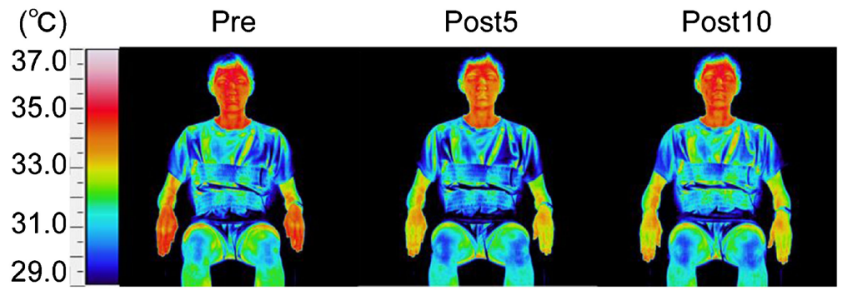

Figure 2. (a) LH (Low-frequency, $0.04-0.15 \mathrm{~Hz}$ ), (b) HF (High-frequency, $0.15-0.45$ $\mathrm{Hz}$, an indicator of PNS), (c) LF/HF (LF/HF ratio indicates, an indicator of SNS), (d) HR (heart rate), (e) BT (body temperature at hands, (f) Visualized image of BT (infrared thermographic instrument). $\bigcirc$ : Natural breathing (Control), $\bigcirc$ : Abdominal Pressure breathing (Pressure), ๑: Abdominal breathing (Abdominal) and ๑: Reverse Abdominal breathing (Reverse). Mean value $\pm \mathrm{SD},{ }^{\star} \mathrm{p}<0.05$. 


\subsection{HR (Heart Rate)}

HR of Control group significantly decreased at Post $5(\mathrm{p}=0.006)$ and Post $10(\mathrm{p}$ $=0.012$ ) compared to that of Pre. Other groups did not show significant differences (Figure 2(d)).

\subsection{BT (Body Temperature)}

Only Pressure group showed significant decreases in BT of both hands. Compared to Pre, BT of Post 5 and Post 10 significantly decreased; the right hand (Post 5, Post 10 and $p=0.002$ and $p=0.001$ ) as well as the left hand (Post 5 and Post 10; $\mathrm{p}=0.001$ and $\mathrm{p}<0.001)$ respectively. At that time, other groups did not indicate such difference (Figure 2(e)).

Visualized image of the change among Pressure group with infrared thermographic instrument is shown as Figure 2(f).

\section{Discussions}

The hypothesis of this study is if traditional breathing methods increase PNS (decrease SNS) and can reduce stress help to reach relax. Our results were the total opposite to it; specific breathing elevated SNS (suppressed PNS). They were understood that specific breathing methods induced stress instead of help to reach relax as we illustrated (Figure 3).

Only Control group showed decrease of HF and HR, showing increase PNS, which indicates the condition of relax, while other groups did not.

At that time, only the Reverse group increased LF/HF ratio as well as only Pressure group showed decreased of BT of both hands. Those results show increase of SNS (decrease PNS = stress). Though specific breathing methods are often recommended to reduce stress and lead to relax, our result showed they induced stress.

We may find three reasons as below. First our subjects might be too young and naive. All of them came across such traditional and specific breathing in front of university professors.

At that time, they have to voluntary breath and must consciously activate $\operatorname{Tr} A$

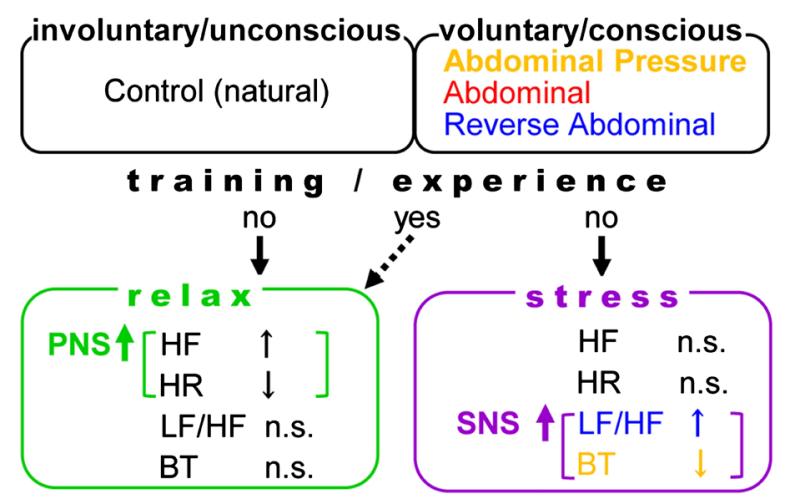

Figure 3. Without enough training/experience specific breathing methods may induce stress instead of help to reach relax. 
muscles in unfamiliar ways, as instructed. In short, those unfamiliar breathing methods gave subjects mental stress. Moreover, it is not difficult to imagine that they feel invisible pressure during experiment. This mental stress might overcome and cancel expected physical effects of traditional breathing, which were expected.

Second, in our subjects, young students did not have enough training or experience. A specific breathing is too difficult to learn oneself; therefore, Nakanishi et al. invented and proposed a program and method for training [5]. In fact, they reported the importance of training and they monitored and confirmed changes in the transverses abdominis muscles thickness by abdominal pressure breathing with ultrasound scanner equipment.

Third, we may find another reason in the duration of breathings. It was limited only one minute [ $(5$ seconds inhale and then 10 seconds to exhale $) \times$ four times] although we chronologically pursued the changes for ten minutes with HR, ANS (LF, HF and LF/HF), and BT as indicators. Effects of one minute breathing might diminish before assessment points.

In Zazen (seated meditation), for example, regular practitioners usually sit for 40 minutes periods while new-comers around 20 minutes [24]. Traditionally, each session lasts the length of time it takes for a stick of incense to burn-around 30 to 40 minutes. Zazen, which has tradition, is passed down through the generations more than eight centuries [25]. Experimentally, it is known that a specific breathing needs 30 to 40 minutes to reduce daily stress to reach relax.

The interest is that only Control group unexpectedly increased PNS level. They simply sat down in armchair. They had minimum mental stress; therefore, their natural breathing using $\operatorname{TrA}$ might indicated maximum physical effects and increased PNS level (= relax). In short, physical action of breathing may be useful to relax in case there is less mental stress.

Although all of our subjects are healthy young male, there might be a lot of individual variation. To overcome this problem, further studies need to be conducted in larger and broader samples. In the further studies, we could provide them appropriate program and enough training time to use to specific breathing. At the same time, we also need to recruit subjects of different age with other background. And then we might obtain other results to compare those of this study.

\section{Acknowledgements}

We thank all volunteers who participated in this study.

\section{Funding}

This work was supported by Grant-in-Aid for Commendatory Research 2019 from Ibaraki Prefectural University of Health Sciences.

\section{Ethical Approval}

This study was approved by the Medical Ethics Committee of Ibaraki Prefectural 
University of Health Sciences (Ibaraki, Japan, 887-R010704).

\section{Conflicts of Interest}

The authors declare no conflicts of interest regarding the publication of this paper.

\section{References}

[1] Editorial (2019) The World Health Organization's Decision about Traditional Chinese Medicine Could Backfire. Nature, 570, 5. https://doi.org/10.1038/d41586-019-01726-1

[2] Kubota, T. (2019) Science and Practice of Tanden (Dantian) Breathing. Pubfull.com.

[3] Muraki, H. (1977) Health Improving Method by Tanden Breathing, Sougensha, ASIN: B01LTIC7U2.

[4] Fuktaki, K. (1911) Abdominal Breathing, Bunseidou-Shinseidou, National Diet Library Digigal Collections. https://dl.ndl.go.jp/info:ndljp/pid/837176/4?tocOpened=1

[5] Nakanishi, T., Tachibana, K., Tomita, K., Shiokawa, M., Mizukami, M. and Imura, S. (2015) Changes in the Transverses Abdominis Muscle Thickness Caused by Abdominal Pressure Breathing. Rigakuryoho Kagaku, 30, 203-206. https://doi.org/10.1589/rika.30.203

[6] Sinderby, C., Navalesi, P., Beck, J., Skrobik, Y., Comtois, N., Friberg, A., Gottfried, S.B. and Lindström, L. (1999) Neural Control of Mechanical Ventilation in Respiratory Failure. Nature Medicine, 5, 1433-1436. https://doi.org/10.1038/71012

[7] Toyoda, H., Hirota, K., Kikuchi, T., Shimazu, D. and Nozaki, Y. (1970) The Role of Interathoratic Pressure (Snatch Pressure) in Various Sports. Activities Journal of Physical Education, 15, 8-16. https://doi.org/10.5432/jipehss.KJ00003396691

[8] Kawabata, M., Shima, N., Hamada, H., Nakamura, I. and Nishizono, H. (2010) Changes in Intra-Abdominal Pressure and Spontaneous Breath Volume by Magnitude of Lifting Effort: Highly Trained Athletes versus Healthy Men. European Journal of Applied Physiology, 109, 279-286. https://doi.org/10.1007/s00421-009-1344-7

[9] Sinderby, C., Navalesi, P., Beck, J., Skrobik, Y., Comtois, N., Friberg, A., Gottfried, S.B. and Lindström, L. (1999) Neural Control of Mechanical Ventilation in Respiratory Failure. Nature Medicine, 5, 1433-1436. https://doi.org/10.1038/71012

[10] Mccarthy, T.A. (1982) Validity of Rectal Pressure Measurements as Indication of Intra-Abdominal Pressure Changes during Urodynamic Evaluation. Urology, 20, 657-660. https://doi.org/10.1016/0090-4295(82)90326-0

[11] Suzuki, S., Toyabe, S., Moroda, T., Tada, T., Tsukahara, A., Iiai, T., Minagawa, M., Maruyama, S., Hatakeyama, K., Endoh, K. and Abo, T. (1997) Circadian Rhythm of Leucocytes and Lymphocytes Subsets and Its Possible Correlation with the Function of the Autonomic Nervous System. Clinical and Experimental Immunology, 110, 500-508. https://doi.org/10.1046/j.1365-2249.1997.4411460.x

[12] Sagiyama, K., Tsuchida, M., Kawamura, H., Wang, S., Li, C., Bai, X., Nagura, T., Nozoe, S. and Abo, T. (2004) Age-Related Bias in Function of Natural Killer T Cells and Granulocytes after Stress: Reciprocal Association of Steroid Hormones and Sympathetic Nerves. Clinical and Experimental Immunology, 135, 56-63.

https://doi.org/10.1111/j.1365-2249.2004.02340.x 
[13] Watanabe, M., Tomiyama-Miyaji, C., Kainuma, E., Inoue, M., Kuwano, Y., Ren, H., Shen, J. and Abo, T. (2008) Role of Alpha-Adrenergic Stimulus in Stress-Induced Modulation of Body Temperature, Blood Glucose and Innate Immunity. Immunology Letters, 115, 43-49. https://doi.org/10.1016/j.imlet.2007.09.010

[14] Kainuma, E., Watanabe, M., Tomiyama-Miyaji, C., Inoue, M., Kuwano, Y., Ren, H. and Abo, T. (2009) Association of Glucocorticoid with Stress-Induced Modulation of Body Temperature, Blood Glucose and Innate Immunity. Psychoneuroendocrinology, 34, 1459-1468. https://doi.org/10.1016/j.psyneuen.2009.04.021

[15] Cannon, W.B. (1929) Organization for Physiological Homeostasis. Physiological Reviews, 9, 399-431. https://doi.org/10.1152/physrev.1929.9.3.399

[16] Bracha, H.S., Ralston, T.C., Matsukawa, J.M., Williams, A.F. and Bracha, A.S. (2004) Does "Fight or Flight” Need Updating? Psychosomatics, 45, 448-449. https://doi.org/10.1176/appi.psy.45.5.448

[17] Abo, T., Watanabe, M., Tomiyama, C. and Kanda, Y. (2014) On/Off Switching of Capillary Vessel Flow Controls Mitochondrial and Glycolysis Pathways for Energy Production. Medical Hypotheses, 83, 99-100. https://doi.org/10.1016/j.mehy.2014.03.035

[18] Watanabe, M., Takano, O., Tomiyama, C., Matsumoto, H., Urahigashi, N., Kainuma, E., Madarame, T., Fukuda, M. and Abo, T. (2012) The Effects of Application of an Ancient Type of Acupuncture Needle on Body Temperature, Immune Function and the Autonomic Nerve System. Health, 4, 775-780. https://doi.org/10.4236/health.2012.410120

[19] Watanabe, M., Takano, O., Tomiyama, C., Matsumoto, H., Kobayashi, T., Urahigashi, N., Madarame, T. and Abo, T. (2012) Skin Rubdown with a Dry Towel, "Kanpu-Masatsu" Is an Aerobic Exercise Affecting Body Temperature, Energy Production, and the Immune and Autonomic Nervous Systems. Biomedical Research, 33, 243-248. https://doi.org/10.2220/biomedres.33.243

[20] Kuusela, T. (2013) Methodological Aspects of Heart Rate Variability Analysis. In: Kamath, M.V., Watanabe, M.A. and Upton, A.R.M., Eds., Heart Rate Variability (HRV) Signal Analysis, CRC Press, Boca Raton, 9-42.

[21] Task Force Report (1996) Heart Rate Variability: Standards of Measurement, Physiological Interpretation, and Clinical Use. Circulation, 93, 1043-1965. https://doi.org/10.1161/01.CIR.93.5.1043

[22] Shaffer, F. and Ginsberg, P. (2017) An Overview of Heart Rate Variability Metrics and Norms. Frontiers in Public Health, 5, 258 https://doi.org/10.3389/fpubh.2017.00258

[23] Uchimura, M., Eguchi, Y., Kawasaki, Yoshii, N., Umeda, T., Takata, M. and Joe, K. (2012) Spatiotemporal Stress Indicator Using LF/HF. Information Processing Society of Japan, MPS-91-2, BIO-32-2.

[24] Nippon.com. “Zazen” (Seated Meditation). https://www.nippon.com/en/features/jg00046

[25] Seisho-ji Temple. Meditate at the Seisho-ji Temple. https://www.serenasystem.com/seisho-ji 